November 4, 2018

Preprint typeset using $\mathrm{LATEX}_{\mathrm{E}}$ style emulateapj v. 08/22/09

\title{
ON THE SURVIVAL OF HIGH-ALTITUDE OPEN CLUSTERS WITHIN THE MILKY WAY GALAXY TIDES
}

\author{
L.A. Martinez-Medina, B. Pichardo, A. Peimbert \& E. Moreno \\ Instituto de Astronomía, Universidad Nacional Autónoma de México, A.P. 70-264, 04510, México, D.F., México; \\ lamartinez@astro.unam.mx \\ November 4, 2018
}

\begin{abstract}
It is a common assumption that high-altitude open clusters live longer compared with clusters moving close to the Galactic plane. This is because at high altitudes, open clusters are far from the disruptive effects of in-plane substructures, such as spiral arms, molecular clouds and the bar. However, an important aspect to consider in this scenario is that orbits of high-altitude open clusters will eventually cross the Galactic plane, where the vertical tidal field of the disk is strong. In this work we simulate the interaction of open clusters with the tidal field of a detailed Milky Way Galactic model at different average altitudes and galactocentric radii. We find that the life expectancy of clusters decreases as the maximum orbital altitude increases and reaches a minimum at altitudes of approximately $600 \mathrm{pc}$. Clusters near the Galactic plane live longer because they do not experience strong vertical tidal shocks from the Galactic disk; then, for orbital altitudes higher than 600 pc, clusters start again to live longer due to the decrease in the number of encounters with the disk. With our study, we find that the compressive nature of the tides in the arms region and the bar have an important role on the survival of small clusters by protecting them from disruption: clusters inside the arms can live up to twice as long as those outside the arms at similar galactocentric distance.
\end{abstract}

Subject headings: galaxies: kinematics and dynamics - galaxies: spiral — galaxies: structure - open clusters and associations: general

\section{INTRODUCTION}

Stars in the disk of our Galaxy seem to have been primarily formed in open clusters. Newly born open clusters originate on the thin molecular gas layer of the Galaxy de la Fuente Marcos \& de la Fuente Marcos 2008); most originate within a galactocentric distance of $10.5 \mathrm{kpc}$ and closer than $180 \mathrm{pc}$ from the disk plane (Dias et al. 2002; Gozha et al. 2012). Less than $10 \%$ survive their embedded stage as gravitationally bound systems (Lada \& Lada 2003); the ones that survive as clusters, with ages ranging between a few million years and approximately $10 \mathrm{Gyr}$, are located between 5 and $20 \mathrm{kpc}$ from the Galactic center. (Freeman 1970; Nagata et al. 1993; Krabbe et al. 1995; Figer et al. 2002; van der Kruit 2002; Paumard et al. 2006; Davies et al. 2012; Moreno et al. 2014; Ramírez Alegría et al. 2014); Albeit the majority are radially confined, and at very low altitudes away from the Galactic disk plane, numerous clusters $(\sim 13 \%$; de la Fuente Marcos \& de la Fuente Marcos 2008) are found at great altitudes; some of those far beyond 200 pc and up to a few kpc.

Interesting scenarios on the origin of highaltitude clusters have been proposed such as: capture from satellite galaxies, formation in situ on high-altitude molecular clouds (Williams et al. 1977; de la Fuente Marcos \& de la Fuente Marcos 2008; Wu et al. 2009; Vande Putte et al. 2010; de la Fuente Marcos et al. 2013), and smoother dynamical intrinsic processes (Quillen 2002; Quillen et al. 2014). Based on the last idea, in a previous paper (Martinez-Medina et al. 2016a), we explored in detail the spiral arms as the mechanism to induce the clusters lifting; this mechanism was disregarded in the past based on studies with simple models of very weak spiral arms (i.e., low amplitude and/or pitch angle). Those models were characterized by having highly idealized potentials, only meant to facilitate analytical calculations to understand better the nature of the spiral arms (Spitzer 1958; Wielen 1977).

In their path through the Galaxy, stellar clusters undergo disruptive stages that dissolve many of them. Lot of work has been devoted to theoretical and observational studies on the evolution and survival of clusters in general -most of them globular clusters - (e.g. Ostriker et al. 1972; Gnedin \& Ostriker 1997; Baumgardt \& Makino 2003; Gieles et al. 2007; Hurley \& Bekki 2008; Küpper et al. 2010; Lamers et al. 2010; Gieles et al. 2011; Kruiissen et al. 2011; Renaud et al. 2011; Berentzen \& Athanassoula 2012; Sánchez-Janssen \& Aguerri 2012; Rieder et al. 2013; Smith et al. 2013; Brockamp et al. 2014; Silva-Villa et al. 2014; Renaud \& Gieles 2015; Rossi et al. 2016). The first disruptive stage, related to two-body relaxation processes, is known as the expansion phase (Takahashi \& Portegies Zwart 2000; Baumgardt \& Makino 2003; Gieles et al. 2011; Madrid et al. 2012). The second stage is known as the evaporation phase (Gieles et al. 2011); in this period the tidal effects of the host galaxy become of great importance for the endurance of clusters as entities; several factors intervene in their evaporation such as the characteristics of the orbit (Baumgardt \& Makino 2003; Webb et al. 2014), depending for example on their distance to the Galactic center (Madrid et al. 2012) and the mass and size of the Galactic disk (Madrid et al. 2014).

In particular, in the case of disk clusters (called open clusters in the Milky Way Galaxy), several contribu- 
tions have to be taken into account to explain the observed short dissolution times. Structures that have been considered to influence the survival expectations include bars, spiral arms (either steady or transient), and molecular clouds (Gieles et al. 2006; Lamers \& Gieles 2006; Gieles et al. 2007; Berentzen \& Athanassoula 2012). For a recent detailed study on the case of the Sun's birth cluster, see Martínez-Barbosa et al. (2016).

The strongest tides encountered by disk clusters occur when they go through high density regions of the disk like giant molecular clouds (Gieles et al. 2006) or spiral arms (Gieles et al. 2007; Kruijssen et al. 2011). One of the strongest tides for massive stellar clusters is the one exerted by the disk for clusters that experiment large vertical excursions (see Gnedin \& Ostriker 1997, for a study on the effect on globular clusters). Although, traditionally, open (or disk) clusters are assumed to have experienced only small excursions away from the Galactic plane, this is not the case for over $10 \%$ of them.

On the other hand, since the 1950's, Oort drew attention to the fact that there is a lack of old open clusters (older than 1 Gyr) in the Galaxy; this cannot be explained by stellar evolution alone, which would make it difficult to observe when they are older. This discrepancy is not only seen in the Milky Way Galaxy, it can also be seen, for example, in the galaxy M51 Gieles et al. 2005; Lamers et al. 2005a). The discrepancy between the number of observed and predicted old open clusters implies that a significant fraction must have been destroyed by different processes (Wielen 1971; Lamers et al. 2005b; Gieles et al. 2007).

The majority of open clusters in the Galaxy, travel as a unit all their (generally short) lifetimes within the thinner disk in cold (approximately gas-like) orbits. For those clusters it is a fact that they cannot be affected by tides produced by the disk because they do not suffer gravitational encounters with the disk. On the other hand, in the case of the oldest open clusters - that tend to be the ones that present the largest excursions away from the plane-, the disk itself is source of important tides able to destroy them as we will show in this work.

Finally, important advances have been done in this field thanks to observational and theoretical dynamical studies (most of them applied to globular clusters). From the theoretical point of view, two important complementary approaches have been employed to simulate a disk galaxy: steady potentials (e.g., Gieles et al. 2014; O'Leary et al. 2014; Cai et al. 2016) and N-body simulations (e.g., Gieles et al. 2006; Rieder et al. 2013; Rossi et al. 2016). While $N$-body simulations provide the only way to study galaxy and clusters evolution selfconsistently, steady potentials are fully adjustable, fast, able to represent a given galaxy (to the best of our observational knowledge of it), and provide orbital details that $N$-body simulations cannot do. Due to the nature of the problem we are tackling we will employ a very detailed steady potential to better represent the Milky Way Galaxy. In this work we focus on the disruption of open clusters; both the formation rate and the initial mass function of those clusters are beyond the scope of this paper.

This paper is organized as follows. In Section 2 we describe the procedure that has been followed to build the Galactic tides of the Milky Way Galaxy model. In
Section 3, the Galactic model, the clusters model, the initial conditions, and the methodology employed are presented. Section 4 shows our results on the survivability of high-altitude open clusters in the Galaxy. A brief discussion is presented in Section 5. Finally, in Section 6. we present our conclusions.

\section{GALACTIC TIDES}

Spatially extended objects such as open clusters, globular clusters or satellite galaxies, are affected by the differential gravitational attraction of their host galaxy (tides). Tidal interactions are described as the spatial derivative of the gravitational force that an object exerts on another. Taking for example an open cluster in a Galactic potential, the acceleration of a star of the cluster at the position $\mathbf{r}$ is given by

$$
\frac{d^{2} \mathbf{r}}{d t^{2}}=-\nabla \phi_{c}(\mathbf{r})-\nabla \phi_{G}(\mathbf{r})
$$

where $\phi_{c}$ and $\phi_{G}$ are the gravitational potentials of the cluster and the host galaxy, respectively.

In order to take the center of mass of the cluster as a frame of reference, one needs to subtract the acceleration of the cluster's center of mass due to the host galaxy. The acceleration of a cluster star at the position $\mathbf{r}^{\prime}$ in this frame of reference is given by

$$
\frac{d^{2} \mathbf{r}^{\prime}}{d t^{2}}=-\nabla \phi_{c}\left(\mathbf{r}^{\prime}\right)-\nabla \phi_{G}\left(\mathbf{r}^{\prime}\right)+\nabla \phi_{G}(\mathbf{0}) .
$$

In this form equation 2 allows to regroup the last two terms, and by considering the case where the distance of the star to the cluster's center of mass is much smaller than the distance of the cluster to the Galactic center $r^{\prime} \ll R_{G}$, it is possible to obtain the linearized equations of motion for a star in the cluster (Renaud et al. 2011) as

$$
\frac{d^{2} \mathbf{r}^{\prime}}{d t^{2}}=-\nabla \phi_{c}\left(\mathbf{r}^{\prime}\right)+\mathbf{T}_{\mathbf{t}}\left(\mathbf{r}^{\prime}\right) \cdot \mathbf{r}^{\prime}
$$

where $\mathbf{T}_{\mathbf{t}}$ is the tidal tensor that contains the effect of the external potential on the cluster, and its components are given by

$$
T_{t}^{i j}\left(\mathbf{r}^{\prime}\right)=\left(-\frac{\partial^{2} \phi_{G}}{\partial x^{\prime i} \partial x^{\prime j}}\right)_{\mathbf{r}^{\prime}} .
$$

Note that the tidal tensor is symmetric $\left(T_{t}^{i j}=T_{t}^{j i}\right)$ and real-valued, so it can be expressed in its diagonal form. Its eigenvalues $\lambda$ measure the strength of the tidal field along the direction of the associated eigenvector. But aside from the strength, the tidal tensor also contains information about the nature of the tides produced by the host galaxy. The tide along a given eigenvector may be compressive $(\lambda<0)$ or extensive $(\lambda>0)$. Moreover, the trace of the tidal tensor is directly related to the local density through Poisson's equation

$$
\operatorname{Tr}(\mathbf{T})=\sum_{i} \lambda_{i}=-\partial^{i} \partial^{i} \phi_{G}=-\nabla^{2} \phi_{G}=-4 \pi G \rho,
$$

where $\rho$ is the local density, which restricts the trace of $\mathbf{T}$ to be $\operatorname{Tr}(\mathbf{T}) \leq 0$, meaning that at least one eigenvalue is negative. 
This restricts the number of scenarios for the nature of the tide. The best known example is the case when the total sum is zero, i.e., there is no external mass inside the volume of interest. In this case, one or two of the eigenvalues will be positive and the tidal field would be extensive at least along one direction, this is called extensive mode. In the scenario we are studying, there will always be Galactic mass inside the cluster's boundary and $\operatorname{Tr}(\mathbf{T})$ will be negative; thus, in addition to the extensive scenarios, there is the possibility for all three eigenvalues to be negative at the same time, meaning that the cluster is compressed along the three directions, this is called a fully compressive mode (Renaud et al. 2009).

\section{GALACTIC MODEL AND NUMERICAL IMPLEMENTATION}

The main goal of this work consists of a statistical study of open clusters destruction, specifically by tides, within a detailed and realistic model of the Milky Way's disk. Such mechanism is effective for clusters that experiment lifts away from the disk plane. Each passing through the disk would be a violent event that may compromise their survival. In our simulations we place several of these objects at different galactocentric radii, different heights above the plane and different initial masses of the clusters. For the purpose of this study, a very detailed Milky Way potential is required, along with a good numerical implementation that is not computationally burdensome for each cluster.

Although $N$-body simulations seem to be the perfect tool to study this type of problems, they are not suitable to achieve the goals of this work yet. First of all, the potential model employed is a very detailed steady model adjusted specifically to the best of recent knowledge of the Galaxy structural and dynamical parameters (i.e., spiral arms and bar angular velocities, masses, density laws, scale-lengths, etc.). Unlike $N$-body simulations, the potential we use is totally adjustable; we are able to fit the whole axisymmetric and non-axisymmetric potential (i.e., spiral arms and bar), in three dimensions to our best understanding of any particular galaxy from observations and models.

Secondly, our model is considerably faster computationally speaking than $N$-body simulations. This allows us statistical studies by swapping the structural and dynamical parameters. At the same time we can study in great detail individual stellar orbital behavior such as: vertical structure, resonant regions, chaotic vs. ordered behavior and periodic orbits to estimate at some degree orbital self-consistency, etc., without having the customary resolution problems of $N$-body simulations. We achieve this without resorting to simple ad hoc models for a spiral perturbation (like the widely employed cosine potential) or the bar (like a Ferrers bar); instead, we employ a three-dimensional (3D) mass distribution for the spiral arms and a bar that fits the Milky Way's density bar, from which we derive their gravitational potential and force fields (for a thorough description of the model see Pichardo et al. 2003, 2004). We introduce briefly here the Galactic model and a detailed description of the numerical implementation to describe open clusters and their interaction with the Galaxy.

\subsection{Milky Way's Gravitational Potential}

The model includes an axisymmetric potential, formed by a Miyamoto \& Nagai (1975) disk and bulge, and a massive halo (Allen \& Santillán 1991). For the spiral arms we employ the PERLAS model (Pichardo et al. 2003) that consists of a bisymmetric three-dimensional density distribution. For the Galactic bar we use a nonhomogeneous triaxial ellipsoid that reproduces the density law of the COBE/DIRBE triaxial central structure of the Galaxy. For further details on the model see Pichardo et al. (2003, 2004).

To fit our model we make use of observational / theoretical parameters from the literature. The length of the bar (semi-major axis) is set to $3.5 \mathrm{kpc}$, with scale-lengths of $1.7,0.64$, and $0.44 \mathrm{kpc}$. The total mass is $1.4 \times 10^{10}$ $\mathrm{M}_{\odot}$ with a pattern speed $\Omega_{B}=45 \mathrm{~km} \mathrm{~s}^{-1} \mathrm{kpc}^{-1}$. For the spiral arms, we consider a pitch angle of $i=15.5^{\circ}$ and a mass ratio of $M_{\text {arms }} / M_{\text {disc }}=0.05$ at a pattern speed $\Omega_{S}$ $=20 \mathrm{~km} \mathrm{~s}^{-1} \mathrm{kpc}^{-1}$. Further details on the parameters of the model and observational restrictions considered were introduced in Table 1 of Martinez-Medina et al. (2016b).

\subsection{Tidal tensor approach}

Regarding the interaction of the cluster with the Galaxy, this can be implemented mainly in two ways: in a direct approach the cluster is placed within the Galactic model, and the acceleration of a star is the sum of the acceleration due to the rest of the cluster and the acceleration due to the Galaxy. Although this approach seems natural, the size of the two systems, cluster and galaxy, differs by orders of magnitude, so that there would be two very different time steps in the simulation. This would increase severely the computation time and may even lead to numerical errors (Renaud \& Gieles 2015). In the tidal tensor approach the equations of motion of a star are solved in the frame of reference of the cluster's center of mass (equation 21). This means that, as seen in Section 2 the contribution of the Galaxy to the equations of motion is contained in the tidal tensor (equation 3). This method, that captures very well the interaction of the cluster with the Galaxy, has been tested in several works by comparing with the direct approach, ensuring its validity and accuracy (Renaud et al. 2011; Rieder et al. 2013).

Since close encounters with individual stars are unlikely to be important and all large structures of the Galaxy have sizes much bigger than the size of a cluster, a linearized tidal tensor approach is ideal (i.e., sufficient and relatively fast) for this study. For our purposes, we need to compute the tides at every point of the precalculated cluster's orbit. The computation of the tidal tensor along the orbit is as described in Section 2. and the total acceleration of every star is the sum of the acceleration due to the rest of the cluster and the tidal acceleration (equation 3).

\subsection{Cluster modeling}

Since we attempt to cover all the possible scenarios that result from different combinations of initial galactocentric radius, orbital height, and cluster's mass, our study requires hundreds of simulations; this compounded with the complexity of the Galactic potential, makes it an impossible task for an $N$-body code. This is because in 
the $N$-body approach particles interact with one another directly, and an accurate representation of the gravitational field will depend on the number of particles, N, which for practical purposes depends on the computational capabilities available. Also the two-body relaxation is a well-known effect unavoidable in $N$-body simulations.

For these reasons, and for the purposes of this work, it is desirable to use a more efficient technique that allows us to approach the gravitational potential of the system without computational restrictions, and isolate the effects of the interaction between the cluster and the Galaxy, avoiding two-body relaxation.

In this work we use the Self-Consistent Field method (SCF) Clutton-Brock 1972, 1973; Hernquist \& Ostriker 1992) to simulate each $\mathrm{N}$ point-mass particle cluster. The method approximates the true gravitational potential and density of the cluster with a finite series of basic functions, i.e., particles in SCF techniques do not interact with one another directly but only through their contribution to the gravitational field of the system.

SCF techniques are truly efficient when the number of basic functions is kept small, which is only possible when the shape of the system does not deviate much from a given symmetry. In the most perturbed scenarios, the gravitationally bound part of stellar clusters may display spheroidal shapes, but for most of the cases they do not deviate significantly from sphericity Combes et al. 1999), mainly because the parts of the cluster more prone to perturbations are the less dense outer regions, instead of the more massive inner region (Lee et al. 2006).

Assuming that the distribution of particles is concentrated, the gravitational potential in the outer parts will be nearly spherical (which is the case for more than half of the open clusters, Nilakshi et al. 2002) and following the SCF technique for the computation of the gravitational potential, we use a basis whose first member is the Plummer density-potential pair (Clutton-Brock 1973); in other words, at each time step in the simulation we account for self-gravity within the cluster, by assuming that the gravitationally bound particles are embedded in a Plummer potential. The criteria to decide the membership of a given star to the cluster is that the star's velocity, $v$, must be less than the local escape velocity, $v_{e}$. Those stars with $v \geq v_{e}$ do not contribute with mass to the cluster at that time step of the simulation (note that the velocity of such star could diminish at a later time, $v<v_{e}$, and it would once again contribute to the mass of the cluster), in this manner the mass of the system evolves with time. Also we let the radial scale in the background Plummer potential to evolve with time by computing, at each time step, the half-mass radius, $r_{h}$, of the distribution, that for the Plummer profile is directly related to the radial scale-length, $a$, by $r_{h} \approx 1.3 a$. In this way the shape of the cluster, although assumed to be always a Plummer sphere, is also evolving with time, with the ability of mimicking expansions and contractions of the system.

For the initial conditions we distribute the particles in space according to a Plummer density profile. The velocity space is populated by using the rejection technique with a Plummer distribution function, in this way the initial distribution of test particles is relaxed with the background potential.
We simulate clusters within galactocentric radii, 5.5 $\mathrm{kpc} \lesssim R_{\min } \lesssim 14 \mathrm{kpc}$, with vertical departures from the disk plane, $0 \mathrm{kpc} \lesssim z_{\max } \lesssim 5.5 \mathrm{kpc}$ and masses, $500 \mathrm{M}_{\odot} \lesssim M_{0} \lesssim 5 \times 10^{4} \mathrm{M}_{\odot}$.

With this numerical approach we do not attempt to reproduce all the physics of the internal mechanisms involved in the evolution of a star cluster; instead, by avoiding the mass loss due to processes intrinsic to the cluster, we focus solely on its interaction with the host galaxy, that in the case of clusters suffering disk shocking is of greater importance in magnitude.

\section{RESULTS}

\subsection{Milky Way's tidal field}

After building the model for the Milky Way's gravitational potential, a computation of the strength of the tides in every point of space will provide us a general insight about the tidal interaction of a given open cluster with the Galaxy, depending on its position, i.e., its orbit through the Galaxy. The tidal field of the model will also give us information about the nature of the tidal modes (extensive or compressive), and of particular interest is the tidal field of the spiral pattern and the central bar.

To compute an $\mathrm{x}-\mathrm{y}$ projection of the tidal field of the model we locate a mesh of $256 \times 256$ points on the plane. The first thing to do in order to obtain the strength of the tide at every point on the mesh, is to compute the nine components of the tidal tensor at every point (equation 4). Since the tidal tensor is given as the second spatial derivative of the potential, which is not totally analytical for our model, we compute its components with a first-order difference scheme. This is done by placing six additional points (two along each direction) around the point of interest, and computing the acceleration at each one of these points. By subtracting the accelerations of every pair of points along the three directions (according to the difference scheme) we obtain the nine components of the tidal tensor on the entire Galactic plane; note that the tensor is expected to be symmetrical but, since the force is analytical and the derivative is numerical, the cross terms are not necessarily identical, in fact they differ in one part in a million, proving the quality of our derivatives. Next, the tensor is diagonalized to obtain the three eigenvalues; its amplitude and sign give us the strength and nature of the tides, respectively.

The upper part of Figure 1 shows the $x-y$ projection of the tidal field measured as $\sqrt{\lambda_{1}^{2}+\lambda_{2}^{2}}$, where $\lambda_{1}$ and $\lambda_{2}$, are the eigenvalues corresponding to the eigenvectors that lay on the Galactic plane. The color map shows that, on the Galactic plane, the tides are stronger near the Galactic center and become weaker at larger radii. By marking the points where all the eigenvalues of the tidal tensor are negative, is interesting to notice that the center of the bar and the center of the spiral pattern, all along the arms, are dominated by fully compressive tides. Also notice that for the spiral arms, when we move along the transversal direction, the tides change from being fully compressive to dropping to zero, then becoming extensive at the border of the arms.

The existence of regions with fully compressive tides within the bar and spiral arms, gives to these structures an important role on the survival of open clusters that move on the Galactic plane. By its nature, the fully 


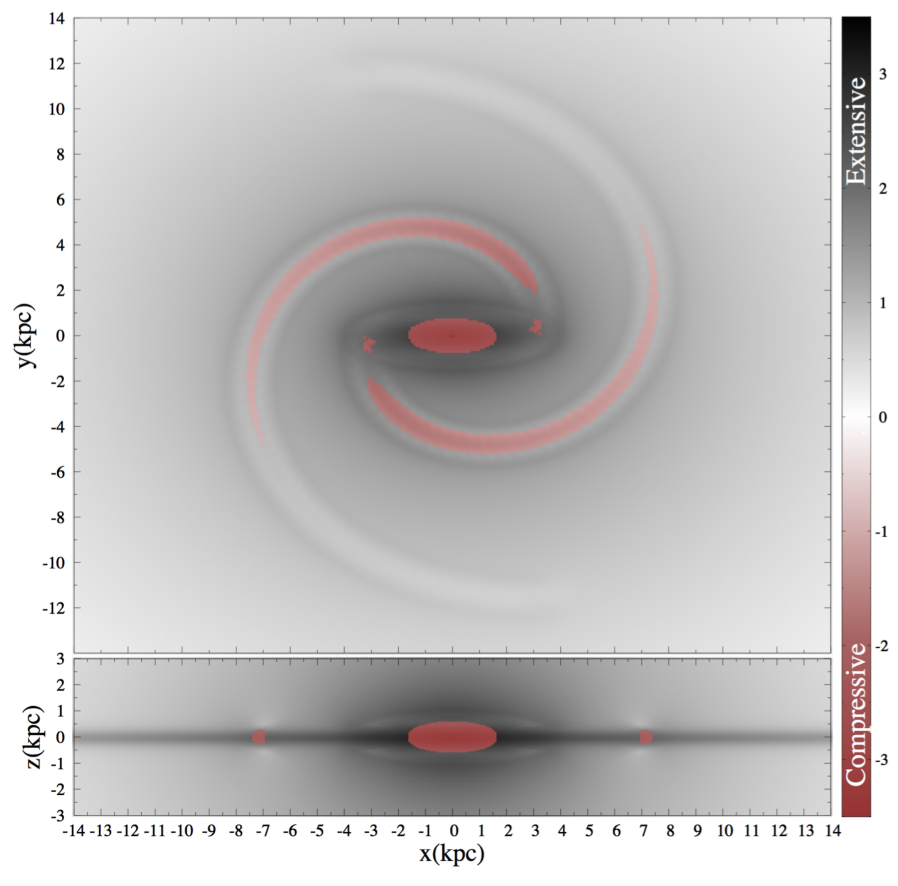

Figure 1. Tidal Field of the Galaxy model. The color map indicates the strength of the tides and the regions with extensive or compressive tidal modes. Compressive regions are located at the center of the bar and the spiral arms.

compressive tides will enhance the binding of the stellar cluster, preventing it from being disrupted as would happen if the cluster moves to other regions of the Galaxy where the tides are extensive; this means that clusters that are born in the spiral arms or the bar will be tidally protected from destruction all the time they stay there. In a preliminary study, we placed several 500 solar mass clusters at a galactocentric distance of $7 \mathrm{kpc}$ at different angular positions; we found that the survival time for those clusters born outside the compressive region of the spiral arms ranged between 34 and $50 \mathrm{Myr}$, while a cluster that was born and lived inside the compressive region of the spiral arm, survived $70 \mathrm{Myr}$, i.e. a cluster inside the compressive region of the arm lived almost twice as long as the ones outside. It should also be noted that the orbit of this cluster only laid inside the compressive region of the spiral arm for $80 \mathrm{Myr}$, but this time depends strongly on the chosen galactocentric radius. In a future work we will present a detailed study of the implications that the fully compressive tidal modes of the bar and spiral arms have on the survival of open clusters in galaxies.

The lower part of Figure 1, is similar to the upper part but it is the $x-z$ projection of $\sqrt{\lambda_{1}^{2}+\lambda_{3}^{2}}$. It shows a transversal cut of the bar and spiral arms, we also see regions of fully compressive tides. But the tidal field is dominated by the extensive modes due to the Galactic disk. In the next sections we will study the interaction of open clusters with the tidal field of the Galaxy, particularly for high-altitude open clusters whose orbits take them to cross the Galactic disk several times.

\subsection{Open Clusters in the Galactic Plane}

The numerical procedure described in Section 3.3 allows us to compute the disruption rates of an open clus- ter in its orbit through the Galaxy: as it moves on the Galactic plane, interacts with the spiral arms, and when its orbit takes it to make vertical excursions above and below the Galactic disk.

The fact that the disruption time depends on the orbital galactocentric distance is a very well known result (Renaud et al. 2011; Berentzen \& Athanassoula 2012). In order to validate our numerical implementation to simulate clusters and isolate their tidal interaction with the Galaxy, we start with open clusters moving in the Galactic plane.

All clusters have an initial half-mass radius $r_{h}=10$ pc. We explore different mean galactocentric radii for the orbit and different initial masses of the cluster.

Figure 2 shows the dependence of the survival time of the cluster on the environment (galactocentric radii) and the initial mass. As anticipated from the tidal map of the Galaxy (Figure 11), the lifetime of all simulated clusters increases as they move away from the Galactic center, this is because the strength of the tides decreases with distance. Regarding the dependence on the initial mass, at all galactocentric radii, clearly the initially more massive clusters will survive longer; some of them even for the age of the Universe if they move in orbits far away from the Galactic center. Also, as a consequence of the small tidal strength at large radii, even non-massive clusters could survive for more than 1 Gyr as long as they move in the outer regions of the disk.

Regarding the clusters that can be totally disrupted, within the lifetime of the Galaxy, by the tidal field produced by the non-axisymmetric structures in the Galaxy, we can try to determine their lifetime. For clusters with $2,000 \mathrm{M}_{\odot} \lesssim M_{0} \lesssim 10,000 \mathrm{M}_{\odot}$ and $5.5 \mathrm{kpc} \lesssim R_{\text {mean }} \lesssim$ $8.5 \mathrm{kpc}$ (all orbits with low eccentricities to assure they lay very close to a chosen galactocentric radius), the survival time can be parameterized as:

$$
\begin{aligned}
\log \tau_{0}\left(R_{\text {mean }}\right)= & -3.1042-0.2628 R_{\text {mean }}+0.4950 \log \left(M_{0}\right) \\
& +0.1268 R_{\text {mean }} \log \left(M_{0}\right)
\end{aligned}
$$

where $\tau_{0}$ is expressed in Gyr, $M_{0}$ in $\mathrm{M}_{\odot}$, and $R_{\text {mean }}$, the mean galactocentric radius is expressed in kpc. This fit has a typical deviation of $1.5 \%$ with our simulations and it is always better than $3.5 \%$.

Intrinsic processes of open clusters may impose further restrictions and better limits to the destruction rates. Our study however, shows the correct behavior of the curves representing the mass loss of the cluster (Figure 2), which adopt a similar shape than those from works using $N$-body models (Takahashi \& Portegies Zwart 2000; Baumgardt \& Makino 2003; Whitehead et al.|2013); i.e., the results of our numerical approach to tidal disruption resemble those of $N$-body codes and give us a good estimation for the survival time of clusters immersed in the tidal field of the Galaxy.

\subsection{High-altitude Open Clusters: Crossing the Galactic Plane}

While preparing this work, an interesting paper recently accepted, employing $N$-body simulations (Rossi et al. 2016) found that for open clusters experiencing large excursions from the Galactic plane, the effect of tides due to crossings with the disk is negligible. However, it should be noticed that, in the majority of 


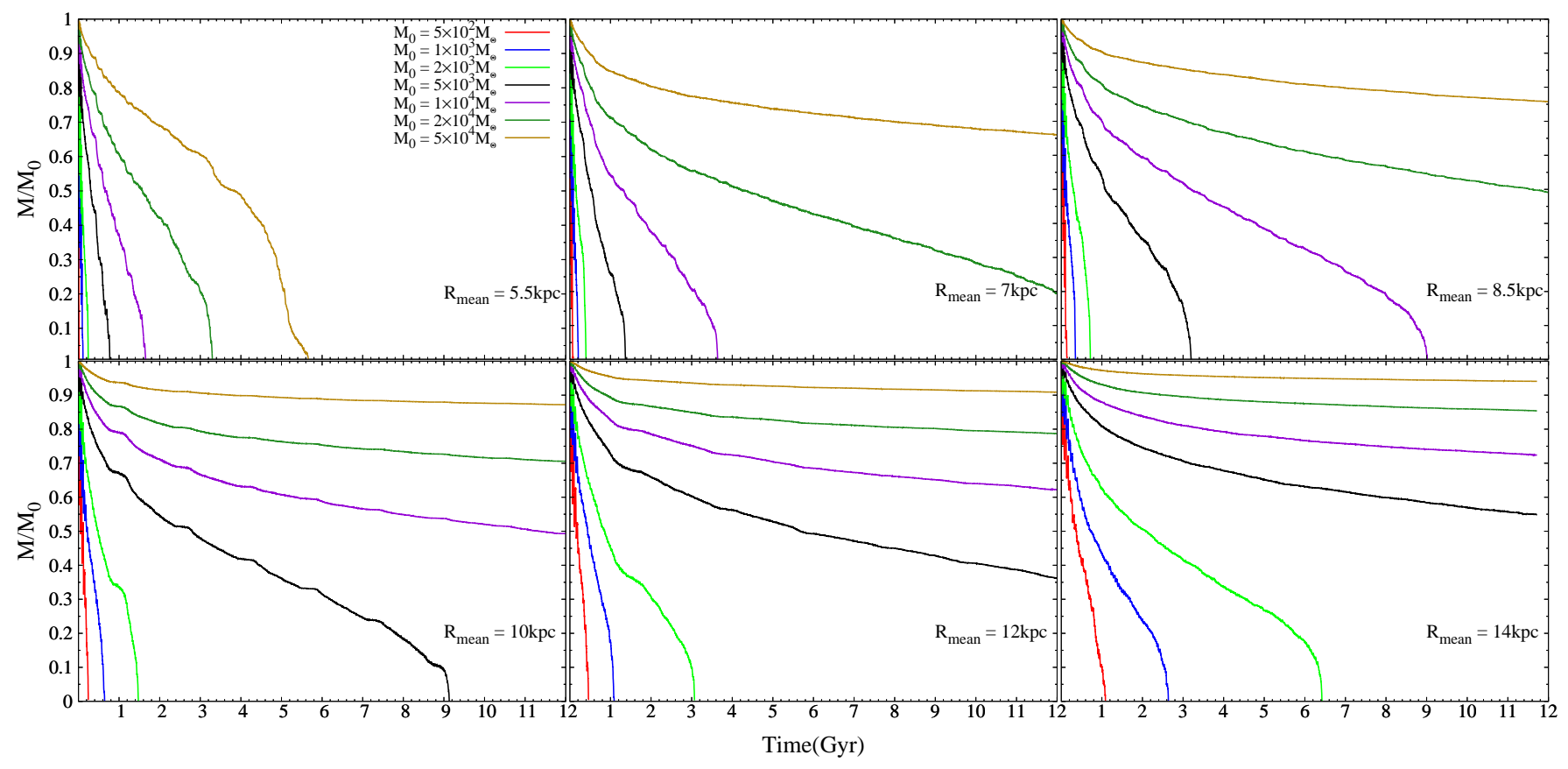

Figure 2. Evolution of the cluster's mass, normalized to its initial value, for different galactocentric radii of the orbit. Each color indicates different initial masses of the cluster, ranging from $500 \mathrm{M}_{\odot}$ to $5 \times 10^{4} \mathrm{M}_{\odot}$.

cases, $N$-body simulations have severe problems to resolve the thin massive disk of galaxies, this means that tidal shocks are likely to be critically underestimated.

Several studies in the literature deal with the evolution of open clusters within a Galactic model. Depending on the mechanisms being studied, which usually are internal to the cluster, the external potential can be simplified as much as needed Gieles et al. 2014; O'Leary et al. 2014; Cai et al. 2016); keeping the external potential simple allows focusing on an specific internal mechanism. Another reason to simplify the galactic potential is the fact that the implementation of a realistic galactic model into an $N$-body code becomes too complicated as well as computationally expensive.

With our adopted numerical implementation to account for the self-gravity of the cluster, and given that the tidal tensor approach captures the contribution of the Galaxy's gravitational field along any orbit, we can place the simulated clusters on more general orbits, no longer restricted to the plane. This method allows us to compare the survival times of clusters moving in the plane of the Galactic disk with those that move above and below, crossing the disk. This will establish the role that the tides due to the disk, which are dominant in the $z$ direction (Figure 1), have on the cluster's disruption.

Upper panel of Figure 3 shows the mass evolution for a cluster initially placed at a galactocentric radii $R=5.5$ $\mathrm{kpc}$ and height $z=600 \mathrm{pc}$ above the Galactic plane. Moving along that orbit, the cluster experiences sudden losses of mass, and its evolution is far from being smooth. Bottom panel of Figure 3 shows the evolution of the cluster's vertical separation from the plane. The color coding, that indicates the strength of the tides at that point of the orbit, shows what we saw in Figure 1 . that the strongest tidal interaction will occur when crossing the disk. By comparing the two panels in Figure 3 we

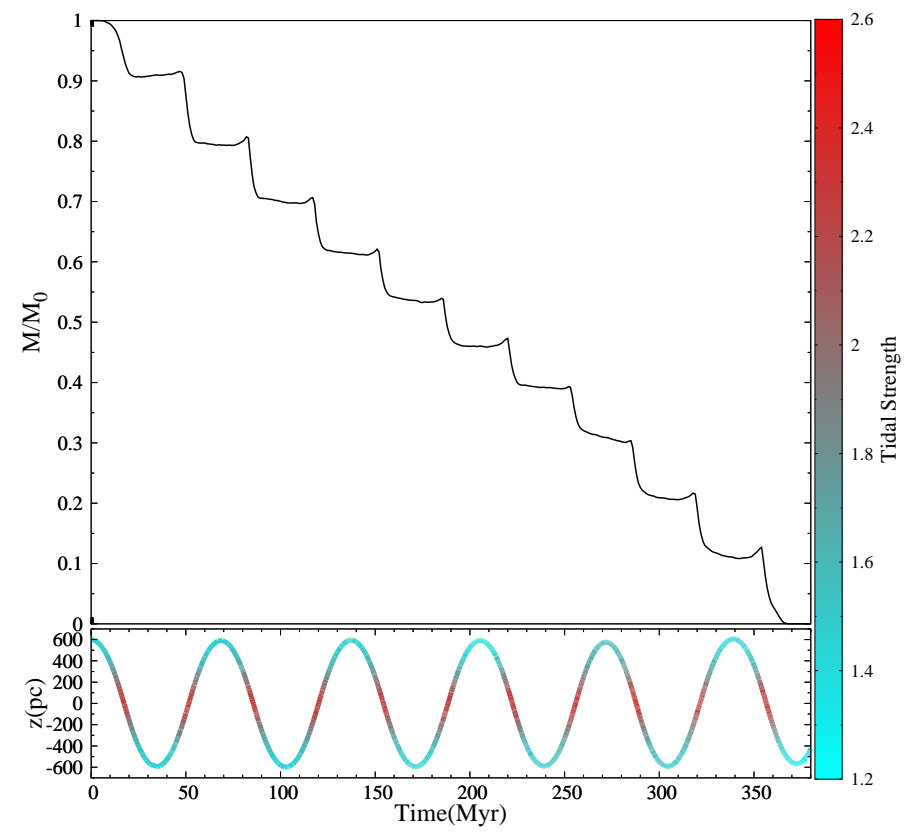

Figure 3. Top: Evolution of the cluster's mass, normalized to its initial value, for a cluster that crosses the plane of the disk several times along its orbit. Bottom: Evolution of the cluster's vertical separation from the plane. The color coding indicates the strength of the tides at that point of the orbit.

can see that every sudden loss of mass occurs just when the cluster crosses the Galactic plane. This result shows how important the vertical displacements are, and that the cluster can easily be disrupted, even when it spends less time interacting with the material of the disk.

We explored several other values of the maximum vertical distance reached by the cluster in order to compare with those that move only in the plane, as well as to 


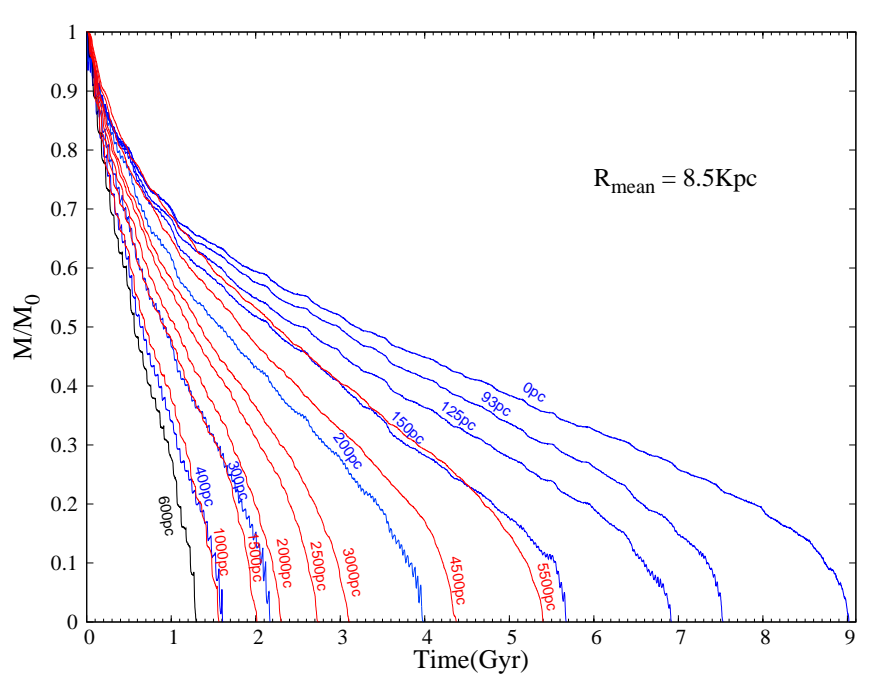

Figure 4. Mass evolution, normalized to its initial value, for clusters moving in different orbits. The maximum orbital height is indicated on each curve and goes from 0 (motion confined to the Galactic plane) to 5500 pc. Blue lines indicate the range of orbital altitudes for which the lifetime of the cluster decreases as the altitude increases. Red lines indicate the regime for which the lifetime of the cluster increases as the altitude of the orbit increases.

quantify how the cluster's survival time depends on its maximum orbital height.

Next we present a series of simulations for clusters placed at different orbits. These orbits differ in the maximum height reached but, for all of them, its projection on the plane has the same $R_{\text {mean }}$.

Figure 4 shows the mass evolution for clusters that reach different orbital heights, ranging from 0 to 5500 $\mathrm{pc}$, all of them at $R_{\text {mean }}=8.5 \mathrm{kpc}$. The evolution of the mass is not equally smooth for all clusters as it depends on the orbital height. Also the lifetime of the cluster varies depending on the orbit.

Figure 4 shows that a cluster survives more time if it is confined to the Galactic plane, without vertical excursions. Then, the lifetime decreases as the orbital height increases. But this trend does not continue forever, it is reversed when the orbital height reaches a turnaround point of about $600 \mathrm{pc}(\sim 2.5$ times the vertical scale of the disk), from there the lifetime of the cluster increases as the orbital height increases. Here we found a correlation between lifetime and orbital height, that exhibits two different behaviors separated by a turnaround height. In the next section we will analyze this result in detail.

\subsection{The Lifetime of High-Altitude Open Clusters}

In Figure 5. we analyze in detail the behavior of the correlation between lifetime and orbital height, derived in the previous section; it is a plot of the maximum orbital height, $z_{\max }, v s$. the lifetime of each cluster, $\tau$ (normalized to the lifetime $\tau_{0}$ of a cluster with the same mass and $R_{\text {mean }}$ that moves on the plane of the Galaxy). We have included a wide exploration of values for the maximum height reached by the orbit, ranging from 0 to 5500 pc, for clusters at two different galactocentric distances and with three different initial masses.

Again, we see that the clusters that survive longer are the ones that move in the plane or remain very close to it, up to $125 \mathrm{pc}(\sim 0.5$ times the vertical scale of the

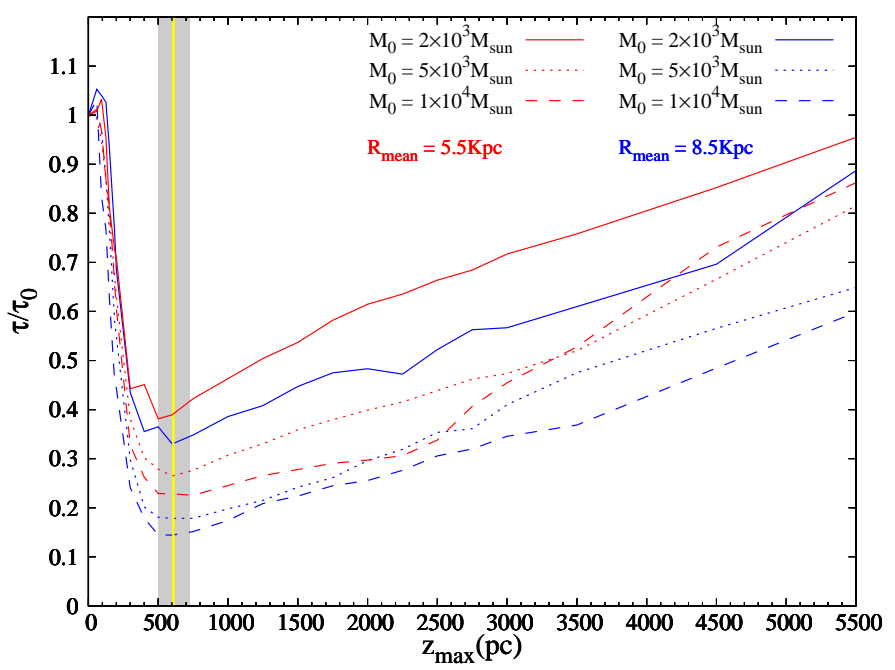

Figure 5. Lifetime of each cluster, $\tau$ (normalized to the lifetime of the cluster that moves on the plane, $\tau_{0}$ ) as a function of the maximum orbital height $z_{\max }$. Red and blue lines correspond to orbits with galactocentric distances of $5.5 \mathrm{kpc}$ and $8.5 \mathrm{kpc}$, respectively. Three different initial masses are presented for each galactocentric radius. The curves reach their minimum survival time at slightly different values of $z_{\max }$, the mean of this values occurs at $z_{\max } \approx 600 \mathrm{pc}$ (yellow line). The shaded band encloses the minima for all the curves.

disk). As the height of the orbit increases, the lifetime decreases until the curve reaches its minimum at some value of $z$, we will call this the turnaround point.

This behavior reveals two distinct regimes in the evolution of clusters whose orbits take them to cross the disk. In the first regime the clusters that live longer are the ones that remain within the denser part of the disk, i.e., those in the plane or with small vertical excursions. Then, for orbits that reach higher altitudes the cluster will cross the disk at a high velocity, which means that the vertical gravitational force acting on the cluster varies rapidly with time, and as a consequence also the tidal strength acting on the cluster. This leads to a considerable loss of bound stars, and hence an important loss of mass each time the cluster crosses the plane of the disk.

In the first regime the lifetime of the cluster starts to decrease rapidly as the crossings through the plane become more violent, then slows down until it reaches a minimum for a given value of $z_{\max }$. From this point, and for higher altitudes, the lifetime of the cluster increases as it raises above the Galactic plane; in this second regime, although the cluster falls towards the disk and crosses it at high velocity, the frequency of its vertical motion diminishes as the orbital height increases, which means that the number of crosses through the Galactic plane is less compared with clusters placed at lower altitudes. Therefore, the cluster interacts less with the tidal field of the disk increasing its lifetime.

The survival time as a function of height away from the plane also depends on the average galactocentric radius and the mass of the cluster. This can be parameterized as:

$$
\frac{\tau(z)}{\tau_{0}}=\left[\alpha+\beta z+\gamma z^{2}\right]+\left[\frac{(1-\alpha)}{1+\exp \left(\frac{z^{1 / 8}-\delta}{0.02544}\right)}\right] ;
$$


Table 1

Parameters used to estimate survival time as a function of height

\begin{tabular}{cccc}
\hline \hline$\chi$ & $\chi_{0}$ & $\chi_{R}$ & $\chi_{M}$ \\
\hline$\alpha$ & 0.21371 & -0.02746 & -0.24042 \\
$\beta$ & 0.07250 & 0.00190 & -0.00539 \\
$\gamma$ & 0.00610 & -0.00168 & 0.00083 \\
$\delta$ & 0.81451 & -0.00146 & -0.0079
\end{tabular}

where the parameters $\alpha, \beta, \gamma$, and $\delta$, have a small dependence on the galactocentric radius and the initial mass of the cluster; the functional form of each parameter can be expressed as

$\chi\left(R_{\text {mean }}, M_{0}\right)=\chi_{0}+\chi_{R}\left(R_{\text {mean }}-7\right)+\chi_{M}\left[\log \left(M_{0}\right)-3.6666\right]$,

where $R_{\text {mean }}$ is in $\mathrm{kpc}, M_{0}$ in $\mathrm{M}_{\odot}$, and the values of the $\chi_{0}, \chi_{R}$, and $\chi_{M}$ are presented in Table 1 . The rms difference between this fit and our simulations is $6.3 \%$.

Notice that the first term in equation 7, represents the second regime (i.e., high velocity, low frequency plane crossing regime); while the first regime is represented by the second part of the equation plus the constant $\alpha$.

In summary, the disruption of open clusters due to the tidal field of the disk will depend on the velocity at which the cluster crosses the Galactic plane and the number of times this occurs. In a first regime, for altitudes between 0 and $600 \mathrm{pc}(\sim 2.5$ times the vertical scale of the disk) the crossing velocity will be the main disruption factor, with slower crosses being gentler. In a second regime, for altitudes higher than $600 \mathrm{pc}$ it will be the number of crosses through the disk, not the velocity, what will determine the lifetime of the cluster. While in the pioneer work by Ostriker et al. (1972), applied to globular clusters (very high altitude case), the destruction of clusters is proportional to the velocity, $v_{z}^{-2}$, while in our simulations, for the second regime, we find that the destruction is nearly independent of the velocity $\left(\propto v_{z}^{-0.2}\right)$ and only proportional to the number of crossings; this is probably related to the fact that clusters in our simulations are moving too slow and are too small for the impulse approximation to be valid.

In any case, for the masses and orbital heights explored in our simulations, the clusters that survive the longest are those that move very close to the plane of the disk.

\subsection{Survival of lifted clusters}

In the previous sections we studied two different survival scenarios for open clusters in the Galaxy: clusters that move all their life in orbits very close to the Galactic plane, or clusters with orbits that oscillate away from the Galactic plane. But, what happens in the transition of clusters from one environment to the other? i.e., what will be the evolutionary path followed by a cluster that is born and lives the first stages of its life in the Galactic plane and afterwards it is lifted by some mechanism above the plane?

In a previous work (Martinez-Medina et al. 2016a), we showed that a mechanism able to lift open clusters way up the Galactic plane are the spiral arms. The presence of the spiral arms induces several clusters to be violently lifted from the Galactic plane, to heights up to $3 \mathrm{kpc}$. An important question for such mechanism is whether the cluster would survive such lifting. For this test, we have selected some high-altitude orbits, i.e., clusters that lived first for a while in the plane and then rose abruptly.

In Figure 6 we show the mass evolution, as well as the orbits, for three examples of lifted clusters from the work by Martinez-Medina et al. (2016a). The blue lines indicate the life stage of the cluster when it moves confined to the Galactic plane; the red lines indicate the second part of its evolution: when the cluster oscillations take it away from the plane, crossing the disk.

Note that the steps in the blue line, in the mass evolution track, are due to the eccentricity of the blue path, while the steps in the red line are not only due to the eccentricity but also due to the crossings of the disk. Nonetheless, in spite of these two disruption factors (orbital eccentricity and disk shocking), we find that several clusters can make the transition from moving in the plane to be located at high altitudes surviving the sudden acceleration associated with the lifting.

\section{DISCUSSION}

It is interesting to note that clusters in the Galactic plane, while moving along the transversal direction of an arm, feel the effect of very different environments in a short period of time: they go from moderately extensive tides (outside the arm), to very extensive, to lightly extensive, to mildly compressive tides (middle of the arm), to lightly extensive, very extensive, and finally to moderately extensive (outside the arm again) in the crossing of an arm.

The compressive nature of the inner region of the arms and the bar has an important role on the survival of small clusters in the disk by enhancing the binding of the clusters, protecting them from disruption: clusters that live in the spiral arm region survive longer within the spiral arms than in any other place of the Galaxy. Additionally, note that colder material of the Galaxy, like new born clusters, would remain longer in the spiral arm region, i.e., an overdensity in the center of the spiral arms of a factor of $4 / 3$ (built in our model from observations, Antoja et al. 2011), results in a slowing down of the clusters, in the spiral arm region, by a factor of $3 / 4$; this means that an overdensity of $36 \%$ (could be even larger in the Milky Way case) represents a slowing down of $1-(1 / 1.36)=27 \%$ by the clusters on their trajectories with respect to the spiral pattern. For us it also means an enhancement of $36 \%$ on the birth rate of the clusters. This implies that a cluster born at $7 \mathrm{kpc}$ inside the spiral arms region, considering the Galactic rotation, shape of the orbits, and arms density contrast, would spend up to 80 million years within the compressive zone of the arm, which means that clusters as massive as $500 \mathrm{M}_{\odot}$ would be more abundant in the spiral arm zone, not only because of the density contrast or because of an enhancement of the star formation, but because of the binding by tides. In a preliminary study, we have calculated the survival time for clusters born outside the compressive region of the spiral arms and found that those are ranged between 34 and $50 \mathrm{Myr}$, while the cluster that was born and lived inside the compressive region of the spiral arm, survived 70 Myr, i.e., the cluster inside the compressive region of the arm lived almost twice as long as the ones outside.

A few mechanisms are known to explain the existence of open clusters at high altitudes away from the disk 


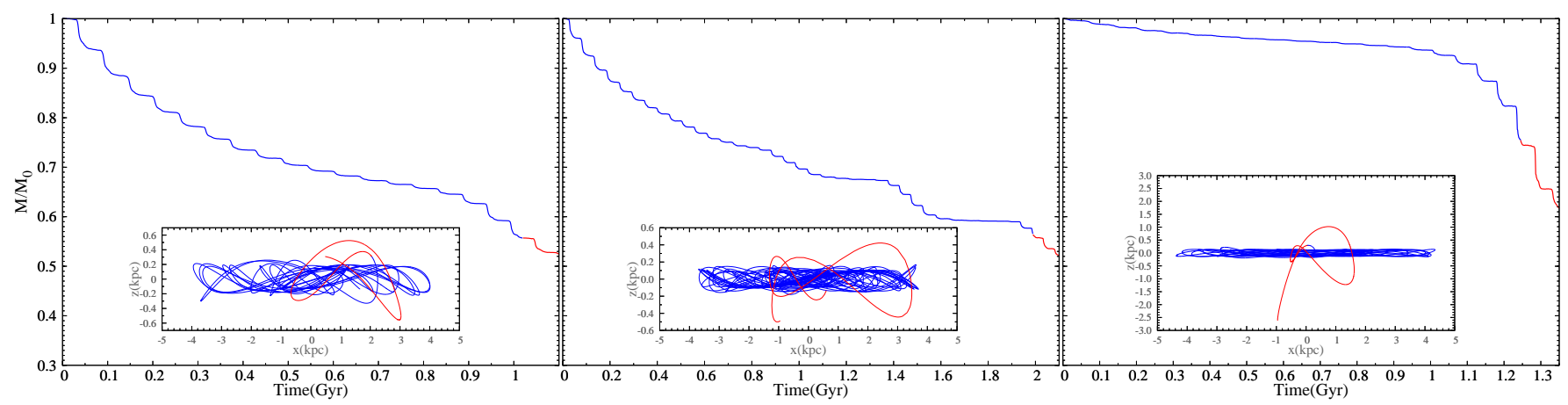

Figure 6. Evolution of the cluster's mass, normalized to its initial value, for clusters lifted above the Galactic plane. The insets show the $x-z$ projection for the orbit of each cluster. The blue path indicates the part of the orbit confined to the plane, while the red path indicates the motion and transition of the cluster into higher altitudes.

plane, but once the clusters are lifted the possibilities of survival are different from the ones in the plane. On one hand, it is assumed that clusters living distant from the Galactic plane have better prospects of surviving as a unit since they live far away from the destructive effects of in-plane substructure, such as molecular clouds, the spiral arms, and the bar. There is, however, a well known destructive mechanism that is, in general, ignored in reference to open clusters: tidal disk shocking; studies in this field ignore it since the majority of open clusters spend all their life as a bound entity in the disk plane, however this is not the case for approximately $13 \%$ of clusters de la Fuente Marcos \& de la Fuente Marcos 2008). On the other hand, some work has found this mechanism to be negligible when studied in $N$-body simulations (Vesperini 1998; Rossi et al. 2016), highly likely due to difficulties to vertically resolve the thin massive Galactic disk. Our approach can in fact resolve the thin Galactic disk (as well as many other components of the Galaxy).

With this technique we find that for high-altitude open clusters, crossing the disk plane can have a severe tidal destructive effect, up to six times as destructive as effects on the disk plane such as the spiral arms and the bar. Two regimes are displayed based on their maximum altitude; in the first one, for clusters with $z_{\max } \lesssim 600 \mathrm{pc}$, the lifetime diminishes as the orbital altitude (as well as the strength of the planar shock) increases, i.e., clusters confined to the Galactic plane would live longer than those with orbits that take them a few hundred parsecs away from the plane because they would not experience vertical tidal shocks from the Galactic disk. In the second regime, for clusters that orbit higher than $\sim 600 \mathrm{pc}$, lifetimes increase again because, although tidal shocks keep being powerful, the frequency of encounters with the disk declines as the altitude increases (i.e., for two orbits with different $z_{\max }$, the number of crossings with the disk, in the same period of time, is different).

Finally, for clusters in the transition from the thin disk to the thick disk, lifted by the effect of the spiral arms, we find that they survive the sudden acceleration.

\section{CONCLUSIONS}

With the use of a very detailed observationally motivated model of the Milky Way Galaxy, we produced an ensemble of numerical simulations of the effect of Galactic tides on open clusters in different regions of the
Galaxy and at different heights away from the disk plane.

We construct a tidal map from our Milky Way model. We find important zones where the tides are fully compressive within the bar and spiral arms. By their nature, fully compressive tides would magnify the binding of stellar clusters, preventing them from being disrupted as fast as it would happen for clusters moving into any other region of the Galaxy.

Fully compressive regions have interesting implications in the evolution of clusters within the Galactic plane, specially on the time small clusters last inside the spiral arms in comparison to other locations of the Galaxy, i.e., small clusters would be more abundant in the spiral arm zone, not only because of the density contrast or because of an enhancement by the star formation, but because of the protecting binding by tides. Preliminary calculations show that clusters inside the compressive region of the arm live almost twice as long as the ones outside.

Contrary to common knowledge, high-altitude open clusters (those over $200 \mathrm{pc}$ ) experience severe tidal destruction when they pass through the disk. In this respect, we find that, up to a certain altitude away from the disk plane (between 0 and $600 \mathrm{pc}$ ), the lifetime of clusters decreases as its maximum orbital altitude increases because tidal shocks with the disk are stronger; clusters confined to the Galactic plane live longer because they do not experience vertical tidal shocks from the Galactic disk (even in the presence of strong arms and bar). For clusters that raise higher than $\sim 600 \mathrm{pc}$, lifetimes increase again, as a function of the maximum altitude, due to the fewer number of encounters with the disk.

We parameterized the survival time for high-altitude open clusters (equation 7), as a function of height away from the plane, average galactocentric radius and the mass of the cluster.

Finally, for clusters in transitional orbits (those that evolve from the thin disk to the thick disk due to the effect of the spiral arms), we find that they experience a sudden acceleration that although violent, is not strong enough to totally destroy them.

We thank the anonymous referee for a careful reading and suggestions that greatly improved this paper. We acknowledge the support of DGTIC-UNAM for providing HPC resources on the Cluster Supercomputer Miztli. We acknowledge DGAPA-PAPIIT through grants IN114114, IN-105916, IN-109716 and IG-100115. L.A.M.M. 
acknowledges support from DGAPA-UNAM postdoctoral fellowship.

\section{REFERENCES}

Allen, C. \& Santillán, A. 1991, Rev. Mexicana Astron. Astrofis., 22,256

Antoja, T., Figueras, F., Romero-Gómez, M., et al. 2011, MNRAS, 418, 1423

Baumgardt, H., \& Makino, J. 2003, MNRAS, 340, 227

Berentzen, I., \& Athanassoula, E. 2012, MNRAS, 419, 3244

Brockamp, M., Küpper, A. H. W., Thies, I., Baumgardt, H., \& Kroupa, P. 2014, MNRAS, 441, 150

Cai, M. X., Gieles, M., Heggie, D. C., \& Varri, A. L. 2016, MNRAS, 455, 596

Clutton-Brock, M. 1972, Ap\&SS, 16, 101

Clutton-Brock, M. 1973, Ap\&SS, 23, 55

Combes, F., Leon, S., \& Meylan, G. 1999, A\&A, 352, 149

Davies, B., de La Fuente, D., Najarro, F., et al. 2012, MNRAS, 419,1860

de la Fuente Marcos, R., \& de la Fuente Marcos, C. 2008, ApJ, $685, \mathrm{~L} 125$

de la Fuente Marcos, R., de la Fuente Marcos, C., Moni Bidin, C., Carraro, G., \& Costa, E. 2013, MNRAS, 434, 194

Dias, W. S., Alessi, B. S., Moitinho, A., \& Lépine, J. R. D. 2002, A\&A, 389, 871

Figer, D. F., Najarro, F., Gilmore, D., et al. 2002, ApJ, 581, 258

Freeman, K. C. 1970, ApJ, 160, 811

Gieles, M., Bastian, N., Lamers, H. J. G. L. M., \& Mout, J. N. 2005, A\&A, 441, 949

Gieles, M., Portegies Zwart, S. F., Baumgardt, H., et al. 2006, MNRAS, 371, 793

Gieles, M., Athanassoula, E., \& Portegies Zwart, S. F. 2007, MNRAS, 376, 809

Gieles, M., Heggie, D. C., \& Zhao, H. 2011, MNRAS, 413, 2509

Gieles, M., Alexander, P. E. R., Lamers, H. J. G. L. M., \& Baumgardt, H. 2014, MNRAS, 437, 916

Gnedin, O. Y., \& Ostriker, J. P. 1997, ApJ, 474, 223

Gozha, M. L., Koval', V. V., \& Marsakov, V. A. 2012, Astronomy Letters, 38, 519

Hernquist, L., \& Ostriker, J. P. 1992, ApJ, 386, 375

Hurley, J. R., \& Bekki, K. 2008, MNRAS, 389, L61

Krabbe, A., Genzel, R. Eckart, A., et al. 1995, ApJ, 447, L95

Kruijssen, J. M. D., Pelupessy, F. I., Lamers, H. J. G. L. M., Portegies Zwart, S. F., \& Icke, V. 2011, MNRAS, 414, 1339

Küpper, A. H. W., Kroupa, P., Baumgardt, H., \& Heggie, D. C. 2010, MNRAS, 407, 2241

Lada, C. J., \& Lada, E. A. 2003, ARA\&A, 41, 57

Lamers, H. J. G. L. M., Gieles, M., \& Portegies Zwart, S. F. 2005a, A\&A, 429, 173

Lamers, H. J. G. L. M., Gieles, M., Bastian, N., et al. 2005b, A\&A, 441, 117

Lamers, H. J. G. L. M., \& Gieles, M. 2006, A\&A, 455, L17

Lamers, H. J. G. L. M., Baumgardt, H., \& Gieles, M. 2010, MNRAS, 409, 305

Lee, K. H., Lee, H. M., \& Sung, H. 2006, MNRAS, 367, 646

Madrid, J. P., Hurley, J. R., \& Martig, M. 2014, ApJ, 784, 95
Madrid, J. P., Hurley, J. R., \& Sippel, A. C. 2012, ApJ, 756, 167

Martínez-Barbosa, C. A., Brown, A. G. A., Boekholt, T., et al. 2016, MNRAS, 457, 1062

Martinez-Medina, L. A., Pichardo, B., Moreno, E., Peimbert, A., \& Velazquez, H. 2016a, ApJ, 817, L3

Martinez-Medina, L. A., Pichardo, B., Moreno, E., \& Peimbert, A. 2016b, MNRAS, 463, 459

Miyamoto, M., \& Nagai, R. 1975, PASJ, 27, 533

Moreno, E., Pichardo, B., \& Velázquez, H. 2014, ApJ, 793, 110

Nagata, T., Hyland, A. R., Straw, S. M., Sato, S., \& Kawara, K. 1993, ApJ, 406, 501

Nilakshi, Sagar, R., Pandey, A. K., \& Mohan, V. 2002, A\&A, 383, 153

O'Leary, R. M., Stahler, S. W., \& Ma, C.-P. 2014, MNRAS, 444, 80

Ostriker, J. P., Spitzer, L., Jr., \& Chevalier, R. A. 1972, ApJ, 176, L51

Paumard, T., Genzel, R., Martins, F., et al. 2006, ApJ, 643, 1011

Pichardo, B., Martos, M., Moreno, E. \& Espresate, J., 2003, ApJ, 582,230

Pichardo, B., Martos, M., \& Moreno, E. 2004, ApJ, 609, 144

Quillen, A. C. 2002, AJ, 124,722

Quillen A. C., Minchev I., Sharma S., Qin Y.-J., Di Matteo P., 2014, MNRAS, 437, 1284

Ramírez Alegría, S., Borissova, J., Chené, A. N., et al. 2014, A\&A, 564, L9

Renaud, F., Boily, C. M., Naab, T., \& Theis, C. 2009, ApJ, 706, 67

Renaud, F., Gieles, M., \& Boily, C. M. 2011, MNRAS, 418, 759

Renaud, F., \& Gieles, M. 2015, MNRAS, 448, 3416

Rieder, S., Ishiyama, T., Langelaan, P., et al. 2013, MNRAS, 436, 3695

Rossi, L. J., Bekki, K., \& Hurley, J. R. 2016, arXiv:1608.02309

Sánchez-Janssen, R., \& Aguerri, J. A. L. 2012, MNRAS, 424, 2614

Silva-Villa, E., Adamo, A., Bastian, N., Fouesneau, M., \& Zackrisson, E. 2014, MNRAS, 440, L116

Smith, R., Sánchez-Janssen, R., Fellhauer, M., et al. 2013, MNRAS, 429, 1066

Spitzer, L., Jr. 1958, ApJ, 127, 17

Takahashi, K., \& Portegies Zwart, S. F. 2000, ApJ, 535, 759

Vande Putte, D., Garnier, T. P., Ferreras, I., Mignani, R. P., \& Cropper, M. 2010, MNRAS, 407, 2109

van der Kruit, P. C. 2002, The Dynamics, Structure \& History of Galaxies: A Workshop in Honour of Professor Ken Freeman, 273,7

Vesperini, E. 1998, MNRAS, 299, 1019

Webb, J. J., Leigh, N., Sills, A., Harris, W. E., \& Hurley, J. R. 2014, MNRAS, 442, 1569

Whitehead, A. J., McMillan, S. L. W., Vesperini, E., \& Portegies Zwart, S. 2013, ApJ, 778, 118

Wielen, R. 1971, A\&A, 13, 309

Wielen, R. 1977, A\&A, 60, 263

Williams, P. M., Brand, P. W. J. L., Longmore, A. J., \& Hawarden, T. G. 1977, MNRAS, 180, 709

Wu, Z.-Y., Zhou, X., Ma, J., \& Du, C.-H. 2009, MNRAS, 399, 2146 